Theorem IV. If one pair of curves

$$
\begin{aligned}
& v=\alpha_{1} u+\alpha_{2} u^{2}+\cdots, \\
& v=\beta_{1} u+\beta_{2} u^{2}+\cdots,
\end{aligned}
$$

is to be equivalent, under the equilong group, to a second pair of curves

$$
\begin{aligned}
& V=A_{1} U+A_{2} U^{2}+\cdots, \\
& V=B_{1} U+B_{2} U^{2}+\cdots,
\end{aligned}
$$

the necessary and sufficient condition is the equality of a single absolute invariant $J$, that is,

$$
J(\alpha, \beta)=J(A, B) .
$$

If the order of contact of the curves of each pair (this is obviously an arithmetic invariant) is $h-1$, the invariant $J$ is of order $2 h-1$.

If $h=1$ (curves not touching), $J$ is the tangential distance $\delta$. If $h=2$ (simple contact), $J$ is a combination of the radii of curvature and their rates of variation, as given above.

Columbia University, NEW YoRK.

\title{
THE INVERSION OF AN ANALYTIC FUNCTION.
}

BY DR. SAMUEL BEATTY.

(Read before the American Mathematical Society, April 28, 1917.)

The demonstration of the existence of the inverse of an analytic function is made to depend in the Weierstrass theory upon the power series representation of the function and in the Cauchy theory upon the Jacobian of the real and imaginary parts of the function with reference to the real and imaginary parts of the variable. The proof presented in the following pages finds its source in the Goursat conception of an analytic function and is related as to method to the theory of sets of points.

Suppose the function $w=f(z)$ exists and has a finite derivative at each point of a simply connected domain $D$. 
Suppose $z_{0}$ is a point of $D$ for which the derivative is different from zero. There is a simple, closed curve $C$ lying entirely in $D$ and containing $z_{0}$ as an interior point, such that for points within or on it the values of the function are all different from one another and the values of the derivative are all different from zero. For if $f(z)-f\left(z^{\prime}\right)=0$ can be satisfied by different values $z, z^{\prime}$ both as near $z_{0}$ as desired, then

$$
\begin{aligned}
f^{\prime}\left(z_{0}\right) & =\lim _{z, z^{\prime} \rightarrow z_{0}} \int_{\Gamma} \frac{f(t) d t}{(t-z)\left(t-z^{\prime}\right)} \\
& =\lim _{z, z^{\prime} \rightarrow z_{0}} \frac{f(z)-f\left(z^{\prime}\right)}{z-z^{\prime}} \\
& =0,
\end{aligned}
$$

the simple closed curve $\Gamma$ lying entirely in $D$ and containing within it $z_{0}$ and the points $z, z^{\prime}$ as above described; while if the derivative equals zero for values $z$ as near $z_{0}$ as desired, then its value at $z_{0}$ is also zero. Denote the aggregate of interior points of $C$ by $\{z\}$ and the aggregate of function values resulting therefrom by $\{w\}$. Denote by $\{z\}^{\prime}$ the derived set of $\{z\}$ and by $\{\alpha\}$ the aggregate of points of $\{z\}^{\prime}$ not in $\{z\}$. Then $\{z\}^{\prime}=\{z\}+\{\alpha\}$. But $\{w\}$ is included in $\{w\}^{\prime}$, for if $\bar{w}$ is an isolated point of $\{w\}$ and if $\bar{z}$ is the corresponding point of $\{z\}$ then $(w-\bar{w}) /(z-\bar{z})$ approaches infinity as $z$ approaches $\bar{z}$, which contradicts the assumption of a finite derivative at $\bar{z}$. Denote by $\{\beta\}$ the aggregate of points of $\{w\}^{\prime}$ not in $\{w\}$. Then $\{w\}^{\prime}=\{w\}+\{\beta\}$.

On the basis of the relation $w=f(z)$ there is a $(1,1)$ correspondence between the points of $\{\beta\}$ and $\{\alpha\}$. For suppose $\alpha$ is a point of $\{\alpha\}$ defined by the sequence $z_{1}, z_{2}, z_{3}, \cdots$ made of points of $\{z\}$ and suppose $\alpha$ is the only limit point of the sequence. The corresponding sequence $w_{1}, w_{2}, w_{3}$, $\cdots$, made up of points of $\{w\}$ must define at least one number $b$. If the latter sequence has more than one limit point, then by dropping out corresponding elements from each sequence two new sequences are secured defining only the numbers $\alpha$ and $b$ respectively. Proceeding from such sequences it appears that

$$
\lim _{n \rightarrow \infty} \frac{w_{n}-f(\alpha)}{z_{n}-\alpha}=f^{\prime}(\alpha) \neq 0, \quad \lim _{n \rightarrow \infty} z_{n}-\alpha=0,
$$


from which it follows that $\lim _{n \rightarrow \infty} w_{n}-f(\alpha)=0$, which associated with the relation $\lim _{n \rightarrow \infty} \vec{w}_{n}-b=0$ furnishes as result $b=f(\alpha)$. It is now evident that there was no possibility of the latter sequence defining more than one number. Hence corresponding to any number $\alpha$ of $\{\alpha\}$ is one and only one number $\beta$ of $\{\beta\}$ so that $\beta=f(\alpha)$. It may be proved in like manner that corresponding to any number $\beta$ of $\{\beta\}$ is one and only one number $\alpha$ of $\{\alpha\}$ so that $\beta=f(\alpha)$. Finally this same method of proof coupled with the fact that $\{\alpha\}$ includes $\{\alpha\}^{\prime}$ may be employed to establish that $\{\beta\}$ includes $\{\beta\}^{\prime}$.

Each point of $\{w\}$ is an inner point of $\{w\}$. For denote the aggregate of all points in the plane not included in $\{w\}+\{\beta\}$ by $\{\theta\}$. Each point of $\{\theta\}$ is an inner point of $\{\theta\}$, since the limit points of $\{w\}+\{\beta\}$ are all included in $\{w\}+\{\beta\}$. It has been proved that no point of $\{w\}$ is a limit point of $\{\beta\}$ and it remains to prove that no point of $\{w\}$ is a limit point of $\{\theta\}$. Suppose that $w$ is any point of $\{w\}$. With $w$ as center draw a circle of radius $\sigma$, within which is no point of $\{\beta\}$. Draw a concentric circle of radius $\sigma / 2$. This latter circle contains within it no points of $\{\theta\}$. For if so, suppose $\bar{\theta}$ is such a point. There is then a circle with $\bar{\theta}$ as center and of radius $\bar{\sigma}<\sigma / 2$ containing within it only points of $\{\theta\}$ and such that any larger concentric circle contains within it points of $\{w\}$ as well. Such circle must have on its boundary a point $\bar{w}$ of $\{w\}$, whose corresponding point is $\bar{z}$ of $\{z\}$. For $z$ sufficiently near to $\bar{z}$, amp $(z-\bar{z})$ can take values differing by any assigned positive number less than $2 \pi$, while amp $(w-\vec{w})$ cannot take values differing by any assigned number greater than $\pi$. Hence amp $[(w-\bar{w}) /(z-\bar{z})]$ is without a limit as $z$ approaches $\bar{z}$, which contradicts the assumption of a derivative at $\bar{z}$, finite and different from zero. An evident extension would serve to show that there are no points of $\{\theta\}$ within the circle of radius $\sigma$.

Moreover, any two points of $\{w\}$ can be joined by a simple, regular curve composed entirely of points of $\{w\}$, for the corresponding points of $\{z\}$ can be joined by a simple, regular curve composed entirely of points of $\{z\}$, which on the basis of the relation $w=f(z)$ furnishes the simple, regular curve desired joining the two points of $\{w\}$. The set of points $\{w\}$ is therefore a domain and the set of points $\{z\}$ is connected therewith by a relation of the form $z=\phi(w)$. The 
fact that $\phi^{\prime}(w)$ exists and equals the reciprocal of $f^{\prime}(z)$ justifies the conclusion that $z=\phi(w)$ is an analytic function of $w$ for the domain $\{w\}$, and this is the inverse of $w=f(z)$ for the domain $\{z\}$.

Suppose next that the derivative is zero at $z_{0}$. It is not possible that

$$
\lim _{z \rightarrow z_{0}} \frac{f(z)-f\left(z_{0}\right)}{\left(z-z_{0}\right)^{n}}=0 \quad(n=1,2,3, \cdots),
$$

unless $f(z)$ is a constant. This is at once evident from the power series representation for $f(z)$, but it is more in line with the subsequent course of the present paper to establish it in the manner now to be described. Where $n-1$ is any assigned positive integer and if

$$
\lim _{z \rightarrow z_{0}} \frac{f(z)-f\left(z_{0}\right)}{\left(z-z_{0}\right)^{n-1}}=0,
$$

define $w_{n-1}(z)$ in $D$ to equal

$$
\frac{f(z)-f\left(z_{0}\right)}{\left(z-z_{0}\right)^{n-1}}
$$

for $z$ different from $z_{0}$ and to equal zero for $z=z_{0}$. Then $w_{n-1}(z)$ is analytic in $D$. If $C$ is any simple, closed curve in $D$ containing $z_{0}$ as an interior point, then for any other interior point $z$

$$
\frac{w_{n-1}(z)}{z-z_{0}}=\frac{1}{2 \pi i} \int_{C} \frac{w_{n-1}(t) d t}{(t-z)\left(t-z_{0}\right)},
$$

from which it follows that

$$
\lim _{z \rightarrow z_{0}} \frac{f(z)-f\left(z_{0}\right)}{\left(z-z_{0}\right)^{n}}=\frac{1}{2 \pi i} \int_{C} \frac{f(t) d t}{\left(t-z_{0}\right)^{n+1}} .
$$

The proposition is, therefore, to show that it is impossible to have

$$
\int_{C} \frac{f(t) d t}{\left(t-z_{0}\right)^{n+1}}=0 \quad(n=1,2,3, \cdots),
$$

unless $f(z)$ is a constant. If $z$ is any interior point other than the center $z_{0}$ of a circle lying entirely within $C$ and if all the integrals above are supposed to be zero, then 


$$
\begin{aligned}
\left(z-z_{0}\right) f\left(z_{0}\right) & =\frac{1}{2 \pi i} \sum_{n=1}^{\infty} \int_{C}\left(\frac{z-z_{0}}{t-z_{0}}\right)^{n} f(t) d t \\
& =\frac{1}{2 \pi i} \int_{C} \sum_{n=1}^{\infty}\left(\frac{z-z_{0}}{t-z_{0}}\right)^{n} f(t) d t \\
& =\left(z-z_{0}\right) f(z) .
\end{aligned}
$$

That is, $f(z)=f\left(z_{0}\right)$, which contradicts the assumption here made that $f(z)$ is not a constant. Hence for a certain positive integral value of $n-1$

$$
\lim _{z \rightarrow z_{0}} \frac{f(z)-f\left(z_{0}\right)}{\left(z-z_{0}\right)^{n-1}}=0, \text { but } \lim _{z \rightarrow z_{0}} \frac{f(z)-f\left(z_{0}\right)}{\left(z-z_{0}\right)^{n}}
$$

either does not exist or if so is not zero. The previous argument shows that this latter limit exists and equals

$$
\frac{1}{2 \pi i} \int_{C} \frac{f(t) d t}{\left(t-z_{0}\right)^{n+1}},
$$

which may be denoted by $\rho e^{i \theta}$. Adopting the definition already given for $w_{n-1}(z)$ it appears that

$$
w_{n-1}^{\prime}\left(z_{0}\right)=\lim _{z \rightarrow z_{0}} \frac{w_{n-1}(z)}{z-z_{0}}=\rho e^{i \theta} .
$$

Substituting $\xi^{n}$ for $w-f\left(z_{0}\right)$, then for any point $z$ of $D$ other than $z_{0}$

Since

$$
\left(\frac{\xi}{z-z_{0}}\right)^{n}=\frac{w_{n-1}(z)}{z-z_{0}}
$$

$$
\lim _{z \rightarrow z_{0}} \frac{f(z)-f\left(z_{0}\right)}{\left(z-z_{0}\right)^{n}}
$$

exists and is different from zero, $z_{0}$ is not a limit point of points $z$ for which $f(z)-f\left(z_{0}\right)=0$. Since for any point $z$ of $D$ other than $z_{0}$

$$
f^{\prime}(z)=\left(z-z_{0}\right)^{n-1}\left[w_{n-1}^{\prime}(z)+(n-1) \frac{w_{n-1}(z)}{z-z_{0}}\right],
$$

$z_{0}$ is not a limit point of points $z$ for which $f^{\prime}(z)=0$, for in that case $w_{n-1}\left(z_{0}\right)$ would equal zero. Hence where $d$ is any fixed positive number less than $\rho \sin (\pi / n)$, a domain $R$ about 
but not inclusive of $z_{0}$ exists so that for any point $z$ therein $\xi^{n}$ and $d w / d z$ are both different from zero, and

$$
\left|\frac{w_{n-1}(z)}{z-z d}-\rho e^{i \theta}\right| \leqq d,
$$

or what is the same thing,

$$
\frac{w_{n-1}(z)}{z-z_{0}}=\rho e^{i \theta}\left(1+\frac{h}{\rho} e^{i \phi}\right),
$$

in which $0 \leqq h \leqq d$. One of the values of $\xi /\left(z-z_{0}\right)$ corresponding is

$$
\rho^{1 / n} e^{i(\theta+2 q \pi) / n}\left(1+\frac{h}{\rho} e^{i \phi}\right)^{1 / n}=\rho^{1 / n} e^{i(\theta+2 q \pi) / n}\left(1+\frac{k}{\rho} e^{i \psi}\right),
$$

in which $0 \leqq k \leqq h$. Hence for such a determination of $\xi /\left(z-z_{0}\right)$ it appears that

$$
\left|\frac{\xi}{z-z_{0}}-\rho^{1 / n} e^{i(\theta+2 q \pi) / n}\right|<\rho^{1 / n} \sin \frac{\pi}{n} .
$$

Therefore, for $z$ in the domain $R$ there are $n$ determinations of $\xi$ so that the corresponding values of $\xi /\left(z-z_{0}\right)$ lie one within each of $n$ equal circles, each circle lying exterior to the remaining $n-1$ circles and the centers of the circles representing the $n$th roots of $\rho e^{i \theta}$. Having regard to the values of $\xi /\left(z-z_{0}\right)$ lying within a definite one, but nevertheless any one of these circles as determined by the values of $z$ in $R$, and noting that $\xi=0$ for $z=z_{0}$, then for the domain composed of $R$ and the point $z_{0}, \xi$ is a one-valued function of $z$. Moreover, the derivative of $\xi$ for $z=z_{0}$ is the number represented by the center of the particular circle chosen. For any point $z$ of $R$

$$
\frac{d \xi}{d z}=\frac{1}{n} \frac{d w}{d z} \xi^{1-n},
$$

which is finite and different from zero. That is, $\xi$ is an analytic function of $z$ for the domain $R$ and the point $z_{0}$, and for this latter point the derivative is not zero. Hence by decreasing $d$, a domain $R$ can be found so that the corresponding values of $\xi$ are all different from one another. Hence corresponding to such a domain $R$ and the point $z=z_{0}$ is a domain $S$ and the point $\xi=0$, the variables of which are connected by the analytic relations 


$$
\xi=\phi(z), \quad z=\psi(\xi) .
$$

It is clear that corresponding to any point $w$ in the vicinity of $f\left(z_{0}\right)$ the function $z=\psi(\xi)$ furnishes $n$ values of $z$. Also the form of $\psi(\xi)$ would depend on the particular circle chosen, but one form may be transformed into any other by replacing $\xi$ by the product of $\xi$ and the appropriate $n$th root of unity.

University of ToRonto.

\section{EMORY MCCLINTOCK.}

But few members of the American Mathematical Society at the present time appreciate the magnitude of the services rendered by its former president, Emory McClintock, who died July 10, 1916.

He was born September 19, 1840, at Carlisle, Pa. His father was the Rev. John McClintock, a learned Methodist Episcopal clergyman, for a time professor of mathematics, Latin, and Greek in Dickinson College, and during the Civil War chaplain of the American Chapel in Paris. He is perhaps best known as the author, with another, of a "Cyclopædia of Biblical, Theological, and Ecclesiastical Literature."

McClintock went to school for the first time at the age of thirteen, and a year later entered the freshman year of Dickinson College. In 1856, when his father left Dickinson College for New York, he entered Yale, and in 1857 he entered Columbia as a member of the class of 1859 . His remarkable ability excited the admiration of his teachers, Professors Charles Davies and William Guy Peck. In April, 1859 in order to meet an emergency caused by the illness of a member of the teaching staff, he was graduated and appointed tutor in mathematics. Soon afterwards his father took charge of the American Chapel in Paris, and in 1860 McClintock resigned his position at Columbia to go abroad. In 1861 he studied chemistry at the University of Göttingen. In 1862 he returned to America with the intention of engaging in the Civil War. He was offered an appointment as second lieutenant of topographical engineers in the United States Army, but on his way to Washington suffered a sunstroke which prevented him 